\title{
Association between psoriasis and ocular disorders: A narrative review
}

\author{
Abdullah Algarni*, Abdullah Almuqrin and Abdulaziz Alarwan \\ Imam College of Medicine, Riyadh, Saudi Arabia
}

\begin{abstract}
Psoriasis is a long lasting immunological assisted skin disease along with many extra cutaneous manifestations, with substantial genetic and environmental influences. It affects about $1.5-3 \%$ of the world's population, though commonly not life threatening, but then again causes significant morbidity and has no remedy. We conducted a systematic search on the sites like Pub Med, Embase, Scopus, and multiple databases. The literature search included MeSH-terms and text words, in the following combination such as Blepharitis, dry eye, psoriasis, uveitis, immunological assisted skin disease. No restrictions were followed in terms of publication dates. The reference lists of included articles were screened for additional references. Psoriatic ocular findings may include conjunctivitis, dry eye, blepharitis, squamous intraepithelial neoplasm of the conjunctiva, corneal abscess and immune-mediated conditions such as uveitis, all of which may lead to articular changes. Its pathogenesis is very complicated and involves many molecules like T cells, APCs, keratinocytes, Langerhans cell, macrophages, NK cells, an array of Th1-type cytokines along with certain growth factors like VEGF, KGF which are instrumental in the development of psoriasis. Moreover, ocular disorders are characterized by a series of interconnected cellular changes in the skin like hyperplasia of epidermal keratinocytes, vascular hyperplasia and ectasia, and infiltration of T lymphocytes, neutrophils, and other types of leukocyte in the affected skin. Generally, ocular complications are managed with nonsteroidal anti-inflammatory drugs, corticosteroids, and immunosuppressive agents. Surgical therapy should be carried out, when there are chances of visual disturbance. Timely and correct diagnosis with quick treatment or referral to an ophthalmologist may prevent systemic and ocular disorders. This review highlights the association of psoriasis and ocular disorders with their clinical sign and symptoms.
\end{abstract}

\section{Introduction}

Psoriasis is a long lasting immunological assisted skin disease along with many extra cutaneous manifestations, with substantial genetic and environmental influences [1]. It affects about $1.5-3 \%$ of the world's population, though commonly not life threatening, but then again causes significant morbidity and has no remedy. The association between the ocular disorder and psoriasis has been documented for years; however, the specific ocular manifestations in psoriasis patients only lately come to light.

Ocular problems of psoriasis are many and occur much later, after the skin involvement; additionally they can upset virtually any part of the eye. These ocular disorders involve the cornea, but may also include the conjunctiva, uvea, sclera, retina, and surrounding structures. Impact of ocular disorders is often neglected and surveys into the quality of life implications of psoriasis, mostly do not give importance to ocular symptoms. Ocular complications are subtle and are every so often unnoticed. Frequent ocular examinations could benefit the psoriatic patients. The commonest ocular manifestations are dry eyes and blepharitis. Others include pinguecula, punctate keratitis, cataract, uveitis, glaucoma and retinal micro-vascular abnormalities.

Hyper proliferation of keratinocytes with abnormal differentiation along with infiltration of inflammatory cells, mainly activated $\mathrm{T}$ cells in the epidermis and papillary part of dermis are common ocular manifestations found in psoriasis patients [2]. Ocular lesions are more common in males and they often occur during psoriasis exacerbations $[3,4]$. The various clinical findings associated with psoriatic eye include, conjunctivitis, dry eye, episcleritis and uveitis. Chandran and his coworkers reported only one ophthalmic abnormality out of $67 \%$ patients with psoriasis, whereas $20 \%$ of them had more than one abnormality. The present figures seem to be higher as postulated by the authors than would be expected; this could be verified only in the presence of a control group or population-based data for comparison [5].

Lambert and Wright in 1976 noted the presence of ocular inflammation with psoriatic arthritis, conjunctivitis and iritis in patients suffering from psoriasis [6]. Moadel and coworkers in 1995 examined a patient with psoriasis associated with a corneal abscess that responded to topical corticosteroids [7]. Karabulut and co-workers evaluated ocular-surface changes and tear-film functions in patients with plaque-type psoriasis. Ocular-surface changes were evaluated on the cell content of the surface conjunctival epithelium by conjunctival impression cytology and tear-film functions by the Schirmer I test and break-up time (BUT). The results showed the early conjunctival changes in patients with psoriasis [8]. Paiva and coworkers in 2000 conducted a study to describe the clinical characteristics of uveitis related to psoriatic arthritis (PsA), and also to compare the uveitis in PsA to the uveitis in spondyloarthropathy (SA) and concluded that uveitis in patients with PsA was more likely to be insidious in onset, continuous, posterior, and active bilaterally compared with uveitis in patients with SA. Patients with uveitis and axial involvement were more likely to be

Correspondence to: Abdullah Algarni, Postal code/zip 13242, Box 7766, Riyadh city - Ghirnatah, Tel: 0112482223: E-mail: A.D.N-08@hotmail.com

Key words: Blepharitis, dry eye, psoriasis, uveitis

Received: February 12, 2017; Accepted: February 26, 2017; Published: February 28,2017 
male and HLA-B27 positive compared with patients with uveitis and peripheral arthritis alone [9]. Paroli and coworkers described a case of bilateral ring-shaped corneal opacity in 30-year-old male patient associated with psoriatic arthritis and ulcerative rectocolitis. The ring shaped corneal lesions may have had an immunological origin [10]. Lima and co-workers in 2012 studied the type and frequency of ocular manifestations in Brazilian psoriatic arthritis (PsA) patients and many ocular abnormalities were found in the PsA patients, including cataracts, glaucoma, and blepharitis. But however, they concluded that keratoconjunctivitis sicca was the most common ocular finding related to psoriatic arthritis [11]. Kilic and co-workers in 2013 performed a study on a total of 100 psoriasis patients and a group of 100 healthy individuals. History was taken from all study and control subjects, and dermatological, systemic, and ophthalmological examinations were performed and Schirmer and BUT values were measured. The number of ocular findings in both eyes in the patient group was found to be statistically higher than that in the control group [12].

Demir and co-workers in 2015 studied 40 patients with chronic plaque-type psoriasis and 40 age- and sex-matched control subjects. The diagnosis of psoriasis was confirmed by skin biopsy. They concluded that retinal sensitivity was affected by the severity of psoriasis vulgaris [13]. Lezcano Carduz VP and coworkers in 2015 reported a case of psoriasis along with along with a rapidly growing nodular lesion with papillomatous characteristics on the bulbar conjunctiva. The patient had a medical history of psoriasis since childhood and psoriatic arthritis since 2009 and had received several immunosuppressive medications for long period. Histological examination of the lesion revealed a squamous intraepithelial neoplasm of the conjunctiva associated with psoriasis [14].

Gonzalez-Andrades and co-workers in 2015 presented two cases of sterile corneal infiltrate secondary to an exacerbation of psoriasis. Both cases were treated by using $0.02 \%$ topical tacrolimus ointment, which resulted in resolution of the symptoms and infiltrates [15]. Sakurai and co-workers reported a case of 27-year-old male patient with panuveitis that was associated with psoriasis vulgaris and was accompanied by retinal vasculitis with retinal neovascularization leading to repeated vitreous haemorrhages [16]. Abbouda and co-workers in 2016 conducted a study on 117 patients in order to compare the clinical features of uveitis in patients affected by psoriasis and psoriatic arthritis (PsA). Later on, they concluded that uveitis in patients with psoriasis and PsA had distinguishing clinical features. PsA patients had more ocular complications than those with psoriasis. [17].

Psoriasis is a common chronic inflammatory cutaneous disease affecting $0.5 \%$ to $2 \%$ of children and youth. This disease affects $4 \%$ of all children below 16 years of age with all types of dermatologic disorders [18]. The various forms of psoriasis have been known thus far; plaque psoriasis (characterized by dry scaly patches), pustular psoriasis (contains pus like fluid mainly infiltrated with white blood cells), erythrodermic psoriasis (characterized by exfoliation of fine scaly skin with pain and itching), guttate psoriasis (characterized by drop like dots), inverse psoriasis (affects the flexure surfaces and characterized by smooth inflamed lesions) and scalp and nail psoriasis.

We conducted a systematic search on the sites like Pub Med, Embase, Scopus, and multiple databases. The literature search included $\mathrm{MeSH}$-terms and text words, in the following combination such as Blepharitis, dry eye, psoriasis, uveitis, immunological assisted skin disease. No restrictions were followed in terms of publication dates. The reference lists of included articles were screened for additional references.

\section{Pathogenesis}

Psoriasis is a hyper-proliferative skin ailment with augmented rates of epidermal turnover. Its pathogenesis is very complicated and involve many molecules like T cells, antigen presenting cells (APCs), keratinocytes, Langerhans cell, macrophages, natural killer (NK) cells, an array of Th1-type cytokines along with certain growth factors like vascular endothelial growth factor (VEGF), keratinocytes growth factor (KGF) which are instrumental in the development of psoriasis [19]. It is an immunologically mediated disease in which activated $\mathrm{T}$ lymphocytes leads to the inflammation in the dermal component followed by epidermal hyperproliferation [20]. A few numbers of mechanisms which are believed to play a critical role in psoriasis are:

$\mathrm{T}$ cell function: $\mathrm{T}$ lymphocytes consist of a functionally distinct population of helper $\mathrm{T}$ cells and cytolytic $\mathrm{T}$ cells. The principal function of $\mathrm{T}$ cells is to recognize the processed peptide antigens that are attached to proteins encoded by the MHC class II genes. Therefore, for activation, T cells need APCs to process and present peptide fragments on the APC cell surface. T cells secrete various lymphokines. T cells may also inhibit immune responses; in this role, these are known as suppressor T cells. Distinct cell membrane proteins are expressed by different populations of T cells. CD4 positivity is shown by most of the helper $\mathrm{T}$ cells while cytolytic and suppressor cells are CD8 positive. Activation of $\mathrm{T}$ cells requires three steps: a binding, $\mathrm{b}$ antigen-specific activation (signal 1), c non-antigen -specific cell-cell interaction (signal 2) $[21]$.

Role of dendritic cells: Dendritic cells serve as a major class of antigen presenting cells found in increased abundance in psoriatic skin lesions [22]. Langerhans cells are a type of immature dendritic cell (iDC) found in normal epidermis and can also be found in psoriasis lesions [23]. iDCs are derived from blood monocytes or other myeloid precursors and have an immunostimulatory role. These iDCs are further stimulated to become mature DCs (mDCs). Psoriasis lesions show a marked increase in dermal DCs. XIIIa and CD11c are expressed by myeloid DCs or iDCs, and CD83 and DC-LAMP proteins are positive for $\mathrm{mDC}$.

Hyperproliferation of keratinocytes: The skin provides a protective mechanism through its multilayered structure. The epidermis consists of five layers, stratum basale, stratum spinosum, stratum granulosum, stratum lucidium, and stratum corneum. Mainly the keratinocytes are formed in the stratum basale and further they migrate towards the stratum corneum. As cells move toward the surface, their organelles disappear and are filled with keratin. The topmost layer of keratin provides a protective feature. In normal conditions the epidermal cell cycle is completed in about four weeks. But in psoriatic skin, the epidermal cell cycle is accelerated. Cell division in the basal layer occurs every 1.5 days, and the migration of keratinocytes to the stratum corneum occurs within approximately four days. This results in hyperproliferation of keratinocytes.

Angiogenesis: Keratinocytes produce pro-angiogenic cytokines (VEGF, IL-8), but the precise mechanism of angiogenesis in psoriasis is still unknown. In psoriasis the endothelial cells swell and become activated these activated endothelial cells migrate, sprout, and lay down a basement membrane with pericytes for structural support to form novel vessel network [24]. This results in widening of the intercellular spaces, and hence, dermal blood vessels dilate thus making it easier for leukocytes to migrate into the skin [25].

Cytokine mediators: In psoriasis, the production of cytokines results 
in the epidermal hyper-proliferation, vascular dilatation and dermal inflammation. The cytokines involved in the development of psoriasis include granulocyte-macrophage colony stimulating factor (GMCSF), epithelial growth factor (EGF), IL-8, IL-12, IL-1, IL-6, IFN- $\gamma$, and TNF- $\alpha$. These cytokines result in keratinocyte proliferation, neutrophil migration, potentiation of Th1 type of responses, angiogenesis, upregulation of adhesion molecules, and epidermal hyperplasia.

Reduced apoptosis: In order to maintain a constant thickness of the epidermis proliferation of keratinocytes in normal epidermis is regulated by apoptotic cell death. The epidermal hyperplasia characteristic of psoriasis is suggested to be due to P53 overexpression and these proliferating cells typically express $\mathrm{Bcl}-2$ that protects them against apoptotic stimuli, while terminally differentiated cells lose Bcl-2 expression [26].

Psoriasis and ocular disorders: Story so far: Psoriasis is a chronic inflammatory disorder which commonly manifests with various extra cutaneous manifestations of which eye involvement is important. Psoriasis may affect the lid, conjunctiva or cornea resulting in the development of ocular manifestations, including conjunctival hyperemia and conjunctivitis, ectropion and trichiasis and corneal dryness with punctate keratitis and corneal melting. Studies done by Chandranet and colleagues and Erbagci and colleagues in turkey had found the prevalence of ocular manifestations in psoriasis to be $67 \%$ and $65 \%$ respectively, which were in congruence with the present study, which showed a prevalence of $70 \%$ [1].

Psoriasis and the eye of patients with psoriasis, uveitis had been commonly thought to occur only in conjunction with psoriatic arthritis, however, there have been many case reports of psoriatic uveitis presenting independent of joint disease. Furthermore, the temporal relationship of these two entities has been disputed. Some recent studies suggest that inflammatory joint manifestations precede uveitis. Nevertheless, some cases of uveitis have been reported to occur even before psoriatic skin disease and uveitis has been reported as the first presenting sign of psoriatic arthritis in $0 \%$ to $11.4 \%$ of cases. The severity of ocular inflammation does not necessarily correlate with extent of joint findings, but may correlate with skin disease [6].

Since the latency period for the development of symptomatic ocular abnormalities may be longer than 5 years, continued surveillance and continued use of appropriate ocular protection by all patients treated with PUVA is indicated [27].

Conjunctivitis: Published articles have suggested conjunctivitis prevalence rates in psoriasis patients as high as $64.5 \%$. Conjunctivitis is a commonly occurring eye condition that can be caused by psoriasis, but it is more commonly due to allergies, bacterial infection, or viral infection.6 Symptoms of conjunctivitis can include redness, tearing, or thick yellow discharge. Conjunctival lesions have been described as demarcated, yellowish-red plaques on the palpebral conjunctiva or as areas of xerotic appearance on the bulbar conjunctiva. Conjunctivitis can lead to xerosis, symblepharon, and trichiasis with further complications involving the cornea [5].

Dry eye (keratoconjunctivitis sicca): Keratoconjunctivitis sicca has been cited at a prevalence rate of $2.7 \%$ of psoriatic arthritis patients. Some studies suggest prevalence rates of dry eyes as high as $18.75 \%$ of psoriasis patients [6].

Episcleritis: Episcleritis (inflammation of the tissue layer covering the sclera) may also occur in conjunction with psoriasis and presents with hyperemia (increased blood flow) that may be pink or even blue, tenderness (although significant tenderness should be cause to suspect scleritis, a more serious condition), and watering [6].

Blepharitis: Psoriasis may affect the eyelids by several means. Cram suggested that blepharitis (common inflammatory condition of the eyelids) is the most common ocular finding in patients with psoriasis. Different symptoms associated with blepharitis such as burning and itching may cause substantial discomfort. The mechanism behind this is not yet clear, but Zengin and his co-workers revealed that augmented epithelial turnover leads to high volumes of cell production and subsequent shedding may ultimately leads to a mechanical block in the Meibomian duct. Blepharitis can be controlled with adequate lid hygiene including warm compresses, eyelid massage and lid scrub traditionally with baby shampoo based formulas [5].

Uveitis: Uveitis is a loose term that refers to a large group of diverse diseases. The International Uveitis Study Group classifies intraocular inflammation into anterior (iris or ciliary body), posterior (choroid or retina), intermediate (vitreum, peripheral retina, and pars plana of the ciliary body), or panuveitis (generalized inflammation of entire uvea) [6].

Uveitis is a potentially serious ocular complication that can occur in patients with psoriasis. Anterior uveitis has been reported to occur in $7 \%$ to $20 \%$ of patients with psoriasis. Uveal involvement tends to be bilateral, prolonged, and more severe. Uveitis can be controlled effectively using corticosteroids and cycloplegic agents with possible adjunct therapy with conventional immunomodulatory therapy. In patients who are refractory to these therapies or cannot tolerate these agents's anti-tumor necrosis factor-alfa therapy is another option.

Psoriatic uveitis may be anterior or posterior or both and thus may require different treatment strategies. Acute anterior uveitis may often be treated with a dilating eye drop to keep the pupil mobile and prevent formation of synechiae (adhesions between the iris and lens). Posterior uveitis, although it may be difficult to appreciate on examination, is more commonly responsible for loss of vision, increasing the urgency for inflammation treatment. Recommended pharmacotherapy has evolved as understanding of the pathogenesis has improved and as specific inflammatory mediators have been identified. Although the traditional treatment has involved corticosteroids or immuno modifying drugs, in recent years, the use of drugs that target the TNF pathway has been suggested for use in the more intractable cases [6]. Uveitis in patients with psoriasis may have distinguishing clinical features [28]. Uveitis is frequently the first indication of a previously undiagnosed HLA-B27associated extraocular disease [29].

Cataract: Lens abnormalities in patients with psoriasis are generally thought to be incidental findings. Chandran et al found that $63 \%$ (63 of 100) of patients had bilateral cataracts although. An association between with corticosteroid or phototherapy use was not found.5 But some case reports have suggested that PUVA therapy in humans may be associated with an increased risk of ocular lens abnormalities, [30] but this is not well established.

Corneal lesion: Corneal involvement in psoriasis is rare and usually secondary to the eyelid or conjunctival complications such as xerosis and trichiasis. The most common presentation is punctuate epithelial keratitis, but lesions can include superficial or deep opacities, stromal infiltrates, neovascularization, erosions, scarring, and even stromal melts [5].

Ophthalmological examination of psoriatic patient: All complaints should be referred to an ophthalmologist for evaluation. Non- 
ophthalmologists can assess a patient's visual acuity and examine the external eye for circumcorneal injection. Referral to an ophthalmologist is essential for definitive diagnosis and treatment [6]. Routine eye examinations are necessary in patients with psoriasis of all types, for early detection of subclinical eye pathologies [31].

\section{Conclusion}

The specific mechanism behind the association of ocular disorders with psoriasis is yet not clear, but it is believed to be multifactorial. Genetic, immunologic, and environmental factors play essential parts in both instigation and maintenance of the inflammatory process. Parallel to some other chronic inflammatory ailments, these factors may modify the presentations of the disease and its clinical course. The eye manifestations in psoriasis can lead to various complications, including vision loss. Psoriatic eye manifestations may proceed to articular changes. These signs and symptoms can be easily neglected by the physician because he least bothers about them. Thus, ophthalmological evaluation must be taken out from time to time in psoriasis patients with ocular symptoms, ensuring an early diagnosis of the ophthalmopathy and suitable treatment with NSAID or immunomodulators, thus preventing loss of visual sensation in patients with psoriasis and ocular disorders. Further studies are needed to assess the association between psoriasis and ocular disorders, including relationships between the stages of the disease and its severity and the impact of treatment, particularly in this country.

\section{Acknowledgments}

Financial support and sponsorship: The author(s) declare that they have no financial competing interests.

Conflicts of interest: The author(s) declare that they have no competing interests.

\section{References}

1. Kolli SR, Boda N, Reddy EA (2016) Ocular manifestations in psoriasis. National Journal of Medical and Dental Research 4: 101-104.

2. Sfikakis PP, Iliopoulos A, Elezoglou A, Kittas C, Stratigos A (2005) Psoriasis induced by anti-tumor necrosis factor therapy: A paradoxical adverse reaction. Arthritis \& Rheumatology 52: 2229-2584.

3. Okamoto F, Umebayasi Y, Ohtsuka F, Hommura S (2001) Factors associated with increased aqueous flare in psoriasis. Jpn J Ophthalmol 45: 172-176. [Crossref]

4. Campanati A, Neri P, Giuliodori K, Arapi I, Carbonari G, et al. (2015) Psoriasis beyond the skin surface: a pilot study on the ocular involvement. International journal of Ophthalmology 35: 331. [Crossref]

5. Rehal B, Modjtahedi BS, Morse LS, Schwab IR, Maibach HI (2011) Ocular psoriasis. J Am Acad Dermatol 65: 1202-1212. [Crossref]

6. Au SC, Yaniv S, Gottlieb AB (2016) Psoriatic eye manifestations. Psoriasis forum 17: 3 .

7. Moadel K, Perry HD, Donnenfeld ED, Zagelbaum B, Ingraham HJ (1995) Psoriatic corneal abscess. Am J Ophthalmol 119: 800-801. [Crossref]

8. Karabulut AA, Yalvac IS, Vahaboglu H, Nurozler AB, Duman S (1999) Conjunctival impression cytology and tear-film changes in patients with psoriasis. Cornea 18: 544548. [Crossref]

9. Paiva ES, Macaluso DC, Edwards A, Rosenbaum JT (2000) Characterisation of uveitis in patients with psoriatic arthritis. Ann Rheum Dis 59: 67-70. [Crossref]

10. Paroli MP, Bruscolini A, De Carlo L, Papale A, Mannino G (2007) Ring keratopathy in a patient with psoriatic arthritis and ulcerative rectocolitis. Ocular Immunology and Inflammation 15: 51-6. [Crossref]

11. Lima FB, Abalem MF, Ruiz DG, Gomes Bde A, Azevedo MN, et al. (2012) Prevalence of eye disease in Brazilian patients with psoriatic arthritis. Clinics (Sao Paulo) 67: 249-253. [Crossref]
12. Kilic B, Dogan U, Parlak AH, Goksugur N, Polat M, et al. (2013) Ocular findings in patients with psoriasis. Int J Dermatol 52: 554-559. [Crossref]

13. Demir HD, Kalkan G, Kurt S, Günes A, Sezer E, et al. (2015) Relationship between retinal sensitivity and disease activity in patients with psoriasis vulgaris. Clinics 70 : 14-17. [Crossref]

14. Carduz VPL, Botín DM, Poveda SPD (2015) Squamous Cell Neoplasm of the Ocular Surface in a Patient with Psoriasis Treated with Tumor Necrosis Factor-Alpha Inhibitors. Cornea 34: 833-840. [Crossref]

15. Gonzalez-Andrades M, Arias-Santiago S, García-Serrano JL, González Gallardo MD McAlinden C (2017) Sterile Corneal Infiltrates Secondary to Psoriasis Exacerbations Topical Tacrolimus as an Alternative Treatment Option. Eye Contact Lens 47: 1-3. [Crossref]

16. Yuri S, Namba K, Mizuuchi K, Nomura T, Ishidaa S (2016) A Case of Severe Panuveitis Associated with Psoriasis Vulgaris Successfully Treated with Infliximab. Case Reports in Ophthalmology 7: 191-194. [Crossref]

17. Abbouda A, Abicca I, Fabiani C, Scappatura N, Peña-García P, et al. (2005) Psoriasis and Psoriatic Arthritis-Related Uveitis: Different Ophthalmological Manifestations and Ocular Inflammation Features. Seminars in Ophthalmology: 1-6. [Crossref]

18. Hajheydari Z, Sarparast L, Shahmohammadi S (2015) Management of psoriasis in children: a narrative review. Journal of Pediatrics Review 3: e131.

19. Das RP, Jain AK, Ramesh V (2009) Current concepts in the pathogenesis of psoriasis. Indian J Dermatol 54: 7-12. [Crossref]

20. Mehlis SL, Gordon KB (2003) The immunology of psoriasis and biologic immunotherapy. J Am Acad Dermatol 49: S44-50. [Crossref]

21. Joshi R (2004) Immunopathogenesis of psoriasis. Indian J Dermatol Venereol Leprol 70: 10-12. [Crossref]

22. Shortman K, Liu YJ (2002) Mouse and human dendritic cell subtypes. Nat Rev Immunol 2: 151-161. [Crossref]

23. McGregor JM, Barker JN, Ross EL, MacDonald DM (1992) Epidermal dendritic cells in psoriasis possess a phenotype associated with antigen presentation: In situ expression of beta 2-integrins. Journal of the American Academy of Dermatology 27: 383-388. [Crossref]

24. Longo R, Sarmiento R, Fanelli M, Capaccetti B, Gattuso D, et al. (2002) Antiangiogenic therapy: Rationale, challenges and clinical studies. Angiogenesis 5: 237256. [Crossref]

25. Creamer D, Sullivan D, Bicknell R, Barker J (2002) Angiogenesis in psoriasis Angiogenesis 5: 231-236. [Crossref]

26. El-Domyati M, Barakat M, Abllel-Razek R (2006) Expression of apoptosis regulating proteins, P53 and Bcl-2, in Psoriasis. Journal of the Egyptian Women's Dermatologic Society 3: 46-51. [Crossref]

27. Stern RS, Thibodeau LA, Kleinerman RA, Parrish JA, Fitzpatrick TB (1979) Risk of cutaneous carcinoma in patients treated with oral methoxsalen photochemotherapy for psoriasis. New England Journal of Medicine 300: 809-813. [Crossref]

28. Durrani K, Foster CS (2005) Psoriatic uveitis: a distinct clinical entity? Am J Ophthalmol 139: 106-111. [Crossref]

29. Monnet D, Breban M, Hudry C, Dougados M, Brézin AP (2004) Ophthalmic findings and frequency of extraocular manifestations in patients with HLA-B27 uveitis: a study of 175 cases. Ophthalmology 111: 802-809.

30. Stern RS (1994) Ocular lens findings in patients treated with PUVA. Photochemotherapy Follow-Up-Study. J Invest Dermatol 103: 534-538. [Crossref]

31. Erbagci I, Erbagci Z, Gungor K, Bekir N (2003) Ocular anterior segment pathologies and tear film changes in patients with psoriasis vulgaris. Acta Med Okayama 57: 299303. [Crossref]

Copyright: (C)2017 Algarni A. This is an open-access article distributed under the terms of the Creative Commons Attribution License, which permits unrestricted use, distribution, and reproduction in any medium, provided the original author and source are credited. 